\title{
The synthesis of travelling ionospheric disturbance (TID) signatures in HF radar observations using ray tracing
}

\author{
A. J. Stocker ${ }^{1}$, N. F. Arnold ${ }^{2}$, T. B. Jones ${ }^{2}$ \\ ${ }^{1}$ Department of Engineering, Leicester University, University Road, Leicester, LE1 7RH, UK \\ E-mail: sto@ion.le.ac.uk \\ ${ }^{2}$ Department of Physics, Leicester University, University Road, Leicester, LE1 7RH, UK
}

Received: 28 September 1998 / Revised: 30 June 1999 / Accepted: 5 July 1999

\begin{abstract}
Characteristic signatures are often observed in HF radar range-time-intensity plots when travelling ionospheric disturbances (TIDs) are present. These signatures, in particular the variation of the F-region skip distance, have been synthesised using a ray tracing model. The magnitude of the skip variation is found to be a function of the peak electron density perturbation associated with the TID and radar frequency. Examination of experimental observations leads to an estimate of the peak electron density perturbation amplitude of around $25 \%$ for those TIDs observed by the CUTLASS radar system. The advantage of using the skip variation over the radar return amplitude as an indicator of density perturbation is also discussed. An example of a dual radar frequency experiment has been given. The investigation of the effect of radar frequency on the observations will aid the optimisation of future experiments.
\end{abstract}

Key words. Ionosphere (auroral ionosphere; ionosphere -atmosphere interactions; ionospheric disturbances)

\section{Introduction}

The importance of gravity waves in the upper atmosphere has been established by many workers (see Hunsucker, 1982; Hocke and Schlegel, 1996, for reviews) since the initial study by Hines (1960). Gravity waves can be generated by disturbances in the auroral current and by instability processes associated with convection electric fields (Robinson, 1994). Quasi-periodic gravity waves may be produced either by dispersive reflections from the Earth's surface (Francis, 1974) or by

Correspondence to: A. J. Stocker periodic variations in the disturbance (Millward, 1994). It is known that energy and momentum can be transferred from the middle atmosphere and from the auroral region to mid-latitudes through the action of gravity waves (Chimonas and Hines, 1970).

Thermospheric gravity waves have often been studied by observing their effects on the ionosphere (e.g. Munro, 1958; Georges, 1968), for example through the use of $\mathrm{HF}$ radio transmitter and receiver systems such as Doppler sounders and ionosondes. Although the relationship between the observations and the underlying waves is complex, sophisticated inversion techniques have provided consistent results (Kirchengast et al., 1995, 1996). Samson et al. (1990) found that the passage of travelling ionospheric disturbances (TIDs), which are associated with atmospheric gravity waves, can cause variations in the power and the Doppler shifts of ground-back-scatter returns from HF radars. Although this work enabled the period and direction of the gravity waves to be derived, their intensity was not found.

The results of an extensive ray tracing simulation of TIDs detected by the Goose Bay HF radar have been reported by Bristow and Greenwald (1995). These authors found that the electron density perturbation amplitude of a TID could be determined by examining the peak ground-scatter power in the radar returns. The ground-scatter power is defined as that which is returned to the radar via a ground or sea reflection rather than by scatter from ionospheric irregularities (e.g. Davies, 1990). The measured powers were compared with those derived at a range of TID perturbation amplitudes from ray tracing. From this study it was concluded that TID electron density perturbation amplitudes in the range 20-35\% were present. In addition, these authors also synthesised a range-time intensity plot from the ray tracing results which reproduced the main features of the TID signatures observed by the radar.

Here a numerical ray tracing model (Jones and Stephenson, 1975) has been employed to simulate the observations made by the CUTLASS HF radar located 
in Finland. Again the effects of TID propagation on the ground-scattered signals observed by the radar have been studied. However, the ray tracing model has also been utilised to investigate the recently reported effect of the passage of a TID on the F-region skip distance, i.e. the closest distance to the radar at which ground-scatter returns are found (e.g. Davies, 1990), observed by the radar (Arnold et al., 1998) rather than the back-scatter power effects modelled by Bristow and Greenwald (1995). It has been found that the variation in the F-region skip distance caused by the passage of a TID is a function of TID electron density perturbation amplitude. The TID perturbation values derived in this fashion are comparable with those derived from the peak radar return power by Bristow and Greenwald (1995) and similar to those which have been observed by EISCAT (e.g. Arnold et al., 1998). However, the modulation of skip distance has the advantage of being observable even when the back-scatter is weak, although it may then be difficult to positively establish the presence of TIDs. The variation in skip distance across the entire radar field of view has also been modelled. A comparison of the simulated results with those obtained by observation yields information as to the direction of travel of the TID phase fronts.

The results of a ray tracing simulation at a range of different radar frequencies are also presented. These results are then compared with preliminary observations obtained during a two-frequency experiment. This study has indicated that while the power at ranges close to the skip diminishes with increasing radar frequency, the variation of the skip increases. The predictions made by the model of the effect of using different radar frequencies will be applied to optimising future experimentation.

\section{Experimental observations}

The CUTLASS HF radar facility has been described by Milan et al. (1997). In brief, it consists of two radars, one located in Finland (see Fig. 1) and one in Iceland, each capable of transmitting in the HF band (roughly 8 $20 \mathrm{MHz}$ ) and each receiving signals which have been scattered from both the ionosphere and the ground (the mode of scatter is distinguished by assuming that ground scattered signals are characterised by small Doppler shifts and a low spectral width). Under normal operation the radar successively transmits and receives in 16 different look directions (or beams) in a fixed, narrow (a few hundred $\mathrm{kHz}$ ), frequency band. For the radar located at Hankasalmi, Finland $\left(62.32^{\circ} \mathrm{N}\right.$, $26.61^{\circ} \mathrm{E}$ ), beam 0 has an azimuth of $-36.3^{\circ}$, beam 15 an azimuth of $12.3^{\circ}$ and beam 11 lies closest to pointing due north. Only data from the Finland station will be discussed here. On 26 January, 1997, the Finland radar operated in a special mode, whereby the transmission frequency was altered as follows. Firstly, a frequency near to $10 \mathrm{MHz}$ was transmitted in the even numbered beams $(0,2, \ldots, 14)$, next a frequency around $12 \mathrm{MHz}$ was employed in the odd numbered beams $(1,3, \ldots, 15)$, then $10 \mathrm{MHz}$ in the odd numbered beams, and finally $12 \mathrm{MHz}$ in the even numbered beams. This cycle was then repeated throughout the experimental period. The dwell time on each beam was $3 \mathrm{~s}$ giving a coverage of all the beams at a given frequency in less than $2 \mathrm{~min}$ (sufficient to resolve the effects of TIDs with periods of greater than $4 \mathrm{~min}$ ). It is important to reiterate that two types of scatter are identified by the radar, namely ionospheric scatter resulting from ionospheric irregularities, and ground-scatter resulting from a reflection of the signal from the ground. It is the latter data which are presented here and in which the signatures marking the passage of TIDs are observed most clearly.

The beam 5 range-time-intensity plots (i.e. groundscatter power as a function of range and time) for both $9.97 \mathrm{MHz}$ and $12.4 \mathrm{MHz}$ during the experimental period (10 to 14 UT) on the 26 January 1997 are presented in Fig. 2. It should be noted that before and after these times there is evidence to suggest that back-scatter was coming from behind the radar (Milan et al., 1997) so these

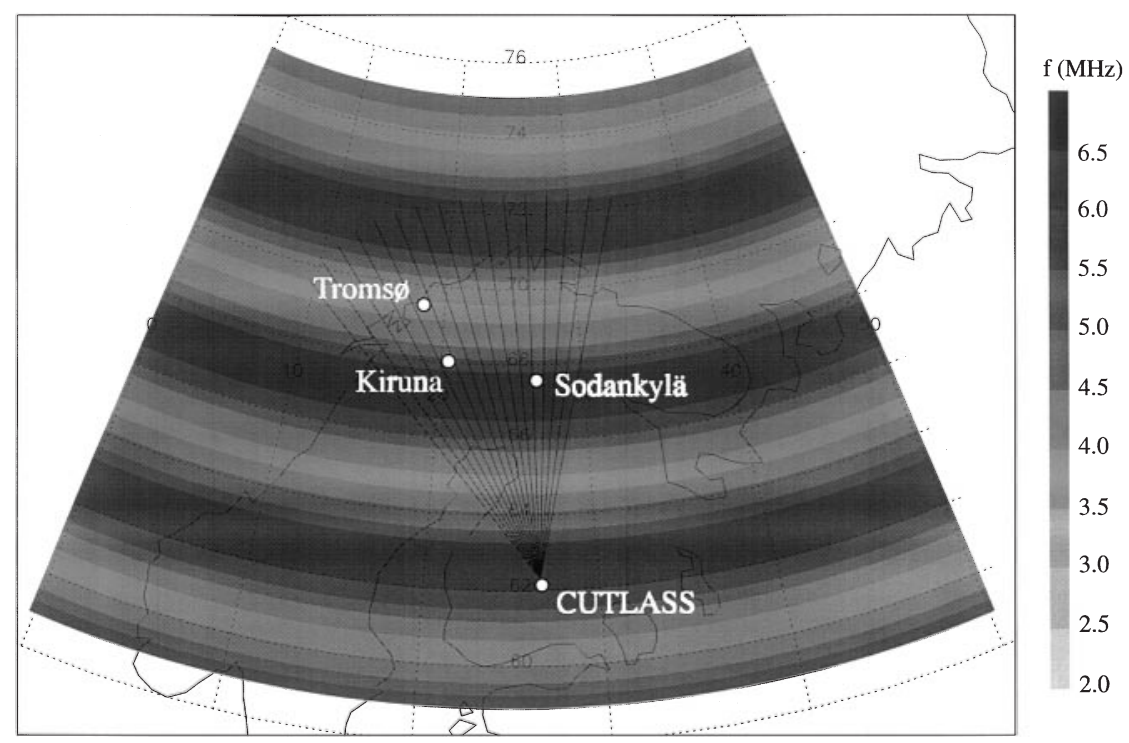

Fig. 1. A map showing the location of the Kiruna, Sodankylä, and Tromsø ionosondes and the CUTLASS (Finland) station. Also shown are the 16 radar beams (at an elevation of $16^{\circ}$ and a frequency of $9.97 \mathrm{MHz}$ ) and the variation in the plasma frequency at a height of $208 \mathrm{~km}$ caused by the model TID 


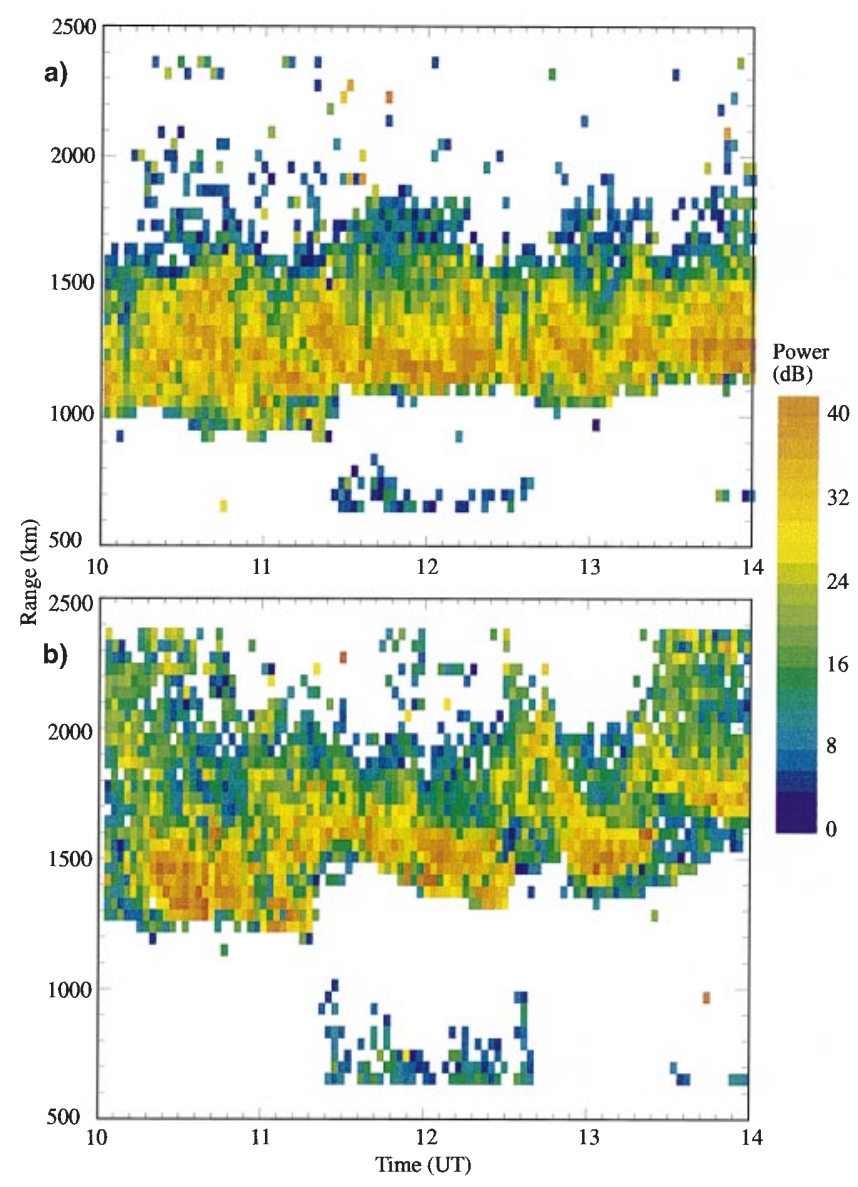

Fig. 2a, b. The ground back-scatter power measured by the CUTLASS Finland radar on 26 January 1997 at frequencies of a $9.97 \mathrm{MHz}$ and b $12.4 \mathrm{MHz}$

periods have been ignored. The interval of interest also exhibits little $\mathrm{E}$ region contamination, so it is reasonably certain that most of the ground-scatter presented is propagated via the $\mathrm{F}$ region. The passage of the TIDs is characterised by two features, firstly the variation in $\mathrm{F}$ region skip distance (i.e. the main set of returns closest to the radar) with time. An example of a strong variation in skip can be found in Fig. $2 b$ where the skip increases from about $1200 \mathrm{~km}$ at $1120 \mathrm{UT}$ to $1500 \mathrm{~km}$ by about 1130 UT before returning to $1300 \mathrm{~km}$ at $12 \mathrm{UT}$. The second feature is the relatively narrow regions of strong back-scatter which approach the radar with time (known as filaments). A good example of such a filament may be observed (Fig. 2b) between 1245 and 1306 UT where a narrow band of strong back-scatter decreases in range from about $2200 \mathrm{~km}$ to just under $1500 \mathrm{~km}$. It is of interest to note that the filaments were quite weak at $10 \mathrm{MHz}$ but moderately well developed at $12.4 \mathrm{MHz}$ since this illustrates an advantage of performing multifrequency experiments. The effect of TIDs on the amplitude of the ground-scatter has been extensively reported in the literature (e.g. Samson et al., 1990; Bristow and Greenwald, 1995).

The variation in $\mathrm{F}$ region skip distance as a function of time and beam number for both radar frequencies is given in Fig. 3. A filter with a band-pass between 15 and $60 \mathrm{~min}$ has been applied to these data. It is important to note that the measurements for each beam are independent and have been treated as such in subsequent analysis. Since there appears to be a reasonable coherence between the different beams, this increases the confidence in these observations. Study of Fig. 3 reveals that essentially the same pattern of variation is observed at both radar frequencies. However, the variation in skip is larger for $12.4 \mathrm{MHz}$ than for $9.97 \mathrm{MHz}$, being about $180 \mathrm{~km}$ in the former case and $150 \mathrm{~km}$ in the latter. Furthermore the variation at high beam numbers tends to lead that at low beam numbers prior to about $13 \mathrm{UT}$, whilst after this time there is little variation with beam number. The results produced by the ray tracing model give a possible interpretation of this effect.

\section{Ray tracing model}

\subsection{Ray tracing code}

The ray tracing results reported have been generated by a modified version of the numerical ray tracing code of Jones and Stephenson (1975). In this study the group range, ground range and launch elevation of rays which reflect from the ionosphere and return to ground are of interest. If the antenna radiation pattern is accounted for, then these data can reveal the number of rays which fall at a given range (either group or ground) from the radar, the separation in range of a ray from its neighbours (the inverse of which is a measure of the ray density), the skip distance (i.e. the range of the closest ray) or the mean launch elevation of rays landing at a given range. The ray density is a measure of the power of the radar signals. The modelled parameters can then be compared with their counterparts in the radar data.

The relative power, $P$ falling at a particular range is calculated from the following expression,

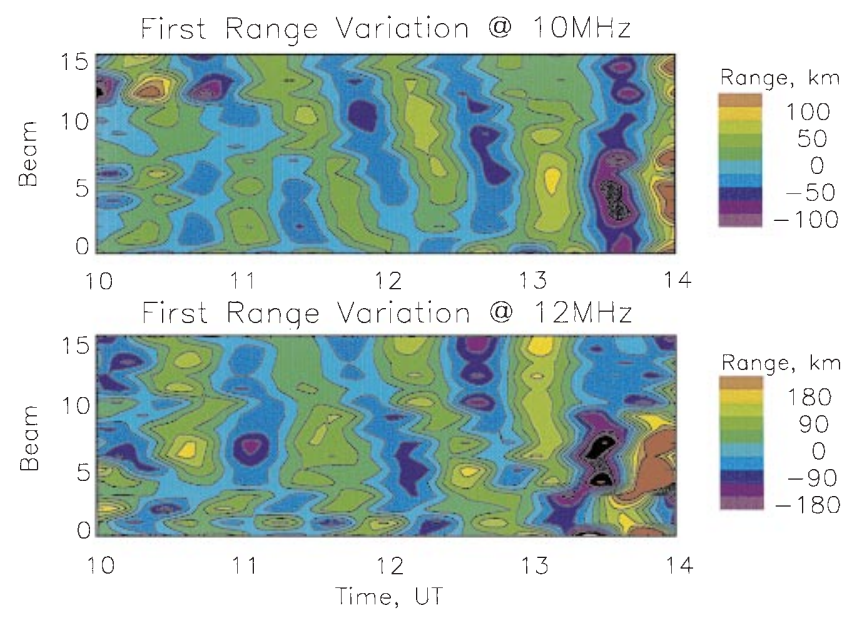

Fig. 3a, b. The skip distance variation measured for all the radar beams at frequencies of a $9.97 \mathrm{MHz}$ and $\mathbf{b} 12.4 \mathrm{MHz}$ 
$P(R) \propto \frac{N_{\text {ray }}^{2}}{2}$

where, $N_{\text {ray }}$ is the number of rays falling into a fixed step range bin. For each of the 16 beams of the radar, only the elevation has been varied in the model with the effect that off great-circle path (GCP) propagation is thereby ignored (although significant off GCP propagation will only occur if large transverse tilts or density gradients are present in the ionosphere). Since the ray-density is varied only in elevation then the rays are effectively carrying amplitude rather than power, hence the $N_{\text {ray }}^{2}$ term in Eq. (1). Furthermore, Eq. (1) assumes that each ray carries the same power and therefore the antenna radiation pattern in elevation must be taken into account, e.g. the number of rays per degree of elevation at boresight is 1.4 times that at the $-3 \mathrm{~dB}$ point of the antenna. Since the radar signals are both transmitted and received by the same antenna array then the effect of the antenna radiation pattern must be applied twice and therefore relative power must be divided by two to reflect this. Additional assumptions implicit in this process are that the principle of reciprocity is obeyed (e.g. Davies, 1990), that the coefficient of ground reflection is the same at all ranges, which is approximately true for the CUTLASS Finland station (Shand et al., 1998), and that the effects of ionospheric absorption can be ignored. It should be noted that the power which results from these calculations is a relative value and not an absolute value.

\subsection{Model parameters}

Since the purpose of the modelling described here is for the results to be compared with experimental observations, it is important to at least approximate the ionospheric conditions prevailing during the experiment. Clearly, given the nature of the high-latitude ionosphere and the large area of coverage (see Fig. 1), a compromise between accuracy and the feasibility of simulating the high-latitude ionosphere must be found. The International Reference Ionosphere (Bilitza, 1990) employed by Bristow and Greenwald (1995), in their ray tracing study is unlikely to be sufficiently accurate at high latitudes. Therefore, ionosonde measurements made simultaneously with the CUTLASS observations have been employed to provide some of the ionospheric parameters required by the ray tracing simulation. The $\mathrm{F}$ region critical frequencies $(f \circ \mathrm{OF})$ observed at three ionosonde stations (Tromsø, Sodankylä, and Kiruna) within the CUTLASS field of view are presented in Table 1. Between 11 and $13 \mathrm{UT}$ the values of $f_{\mathrm{OF}} 2$ are similar at each of the three stations. At 10 and 14 UT there appears to be a gradient in both the north-south and east-west directions. Clearly at these times the assumption made in the modelling that no off great circle propagation occurs is likely to be invalid. It should be noted that while data were obtained at hourly intervals at Sodankylä and Kiruna, at Tromsø ionograms were recorded every $12 \mathrm{~min}$. There is some
Table 1. F-region critical frequencies $(\mathrm{MHz})$ observed by three ionosondes in the field of view of the Finland station of the CUTLASS radar on 26 January, 1997

\begin{tabular}{lccccc}
\hline Station & \multicolumn{4}{l}{ Time (UT) } \\
\cline { 2 - 6 } & 10 & 11 & 12 & 13 & 14 \\
\hline Tromsø & 4.2 & 4.8 & 4.9 & 4.4 & $-^{\mathrm{a}}$ \\
Sodankylä & 4.9 & 4.9 & 4.9 & - & 2.2 \\
Kiruna & 5.9 & 5.0 & 5.3 & 4.6 & 3.2 \\
\hline
\end{tabular}

${ }^{\mathrm{a}}$ At 13:28 UT, foF 2 was $2.5 \mathrm{MHz}$

temporal variation in the critical height of the F2-layer $\left(h_{\mathrm{mF} 2}^{\prime}\right)$ measured at Tromsø quite possibly related to the presence of the TIDs observed by the CUTLASS radar in addition to the expected diurnal variation. For the modelling, the $\mathrm{F}$ region critical frequencies measured at Sodankylä were chosen since this station lies closest to the mid-point of skip-distance rays (see Fig. 1) and because the $f_{\mathrm{OF}} 2$ values remain constant for the period 10-12 UT. The electron density profile employed for the modelling was of simple double Chapman-layer type (model DCHAPT in Jones and Stephenson, 1975). The other parameters required in this model (critical height, $h_{\mathrm{mF} 2}$, and scale height, $H_{\mathrm{F} 2}$ ) were obtained by ensuring that the skip distance returned by ray tracing (with no TID present) was within half of a range gate $(22.5 \mathrm{~km})$ of that observed by the CUTLASS radar at both of the radar frequencies employed (namely 9.97 and $12.4 \mathrm{MHz}$ ). The value of $h_{\mathrm{mF} 2}$ was further constrained to be less than the critical virtual height, $h_{\mathrm{mF} 2}^{\prime}$, observed by the ionosonde at Sodankylä. Neither tilts nor gradients have been included in the electron density model. Although it is clear from Table 1 that such an electron density model cannot accurately represent the conditions over the entire radar field-of-view for the whole experimental interval, such an approximation has the merit of ease of implementation and, as will be seen, yields model results which are reasonably consistent with observations. It should also be noted that the effects of the geomagnetic field and collisions have also been neglected, and that only one-hop propagation paths have been considered.

The TID model employed is described in Jones and Stephenson (1975) as the "WAVE" model. For completeness a brief description of the model is given below. The modified electron density, $N(z, \theta, \phi)$ is given by,

$N=N_{0}(1+\Delta)$

where $N_{0}$ is the unperturbed electron density and $\Delta$ is given by the following equation,

$$
\begin{aligned}
\Delta=\delta & \cdot \exp \left\{-\left[\frac{\left(z-z_{0}\right)}{H}\right]^{2}\right\} \\
\cdot & \cos \left\{2 \pi\left[t^{\prime}+\left(\frac{\pi}{2}-\theta\right) \frac{R_{0}}{\lambda_{x}}+\frac{z}{\lambda_{z}}\right]\right\}
\end{aligned}
$$

where $\delta$ is the peak perturbation amplitude (as a fraction of $N_{0}$ ) of the wave, $z$ is the height, $z_{0}$ is the height of maximum wave amplitude, $H$ is the wave amplitude 
"scale height", $t$ ' is the time in wave periods (effectively the phase of the TID), $\theta$ is the colatitude, $R_{0}$ is the radius of the Earth, and $\lambda_{x}$ and $\lambda_{z}$ are the horizontal and vertical wavelengths, respectively. Note that the perturbation is independent of longitude $\phi$, and the irregularity "travels" from the North Pole to the South Pole (i.e. the phase fronts move southwards with increasing $t^{\prime}$ ).

Values of these parameters which have been employed in the modelling process and which are consistent with those for a strong medium-scale TID are given in Table 2. A map of the variation of $f_{0}$ at a height of $208 \mathrm{~km}$ (corresponding to the $h_{\mathrm{mF} 2}$ observed at Sodankylä) caused by such a model TID is presented in Fig. 1. The rays launched in each of the radar's sixteen beam directions which land close to the skip distance are also marked on the map.

\subsection{Model results}

A method for determining the signal intensity as a function of range from the ray tracing results was outlined earlier. An RTI plot similar to those obtained by the radar can then be constructed by running the ray tracing for different TID phase values ( $t^{\prime}$ in Eq. 3), i.e. essentially simulating the passage of time, and combining the results. Examples of such plots, where the time axis has been replaced by TID phase and where each panel represents a different value of $\delta$ are given in Figs. 4 and 5. It must be remembered that because of the cyclical nature of the TID model employed in the ray tracing, the results at $t^{\prime}=0 \%$ are the same as those at $t^{\prime}=100 \%$. There are a number of features present in Figs. 4 and 5 which are worth noting. Firstly, narrow regions of strong back-scatter (i.e., filaments), similar to those observed by the radar (Fig. 2), are present for values of $\delta$ of $15 \%$ and greater. Next, for $9.97 \mathrm{MHz}$ the filament splits into two at values of $\delta$ greater than $15 \%$, while at $12.4 \mathrm{MHz}$ there are two strong and one weak filament at $25 \%$ and three at $35 \%$, although the third is somewhat weaker than the other two. The filaments have been attributed to the presence of concave reflection surfaces in the ionosphere (Samson et al., 1990; Bristow and Greenwald, 1995). Finally, there is clearly a variation of skip distance during the passage of the TID, a subject which is discussed in more detail later. The principal features of these results are in broad agreement with those obtained by the model of Bristow and Greenwald (1995).

The ray tracing model provides the ground range of the ray which lands closest to the radar, i.e. the skip distance. This has then been plotted as a function of time (i.e. TID phase) and TID perturbation amplitude in Fig. 6. Examination of this figure and Fig. 7, where the

Table 2. Model TID parameters

\begin{tabular}{lllll}
\hline$h_{\max }(\mathrm{km})$ & $\delta(\%)$ & $L_{\mathrm{h}}(\mathrm{km})$ & $L_{v}(\mathrm{~km})$ & $H(\mathrm{~km})$ \\
\hline 208 & 20 & 500 & 100 & 60 \\
\hline
\end{tabular}

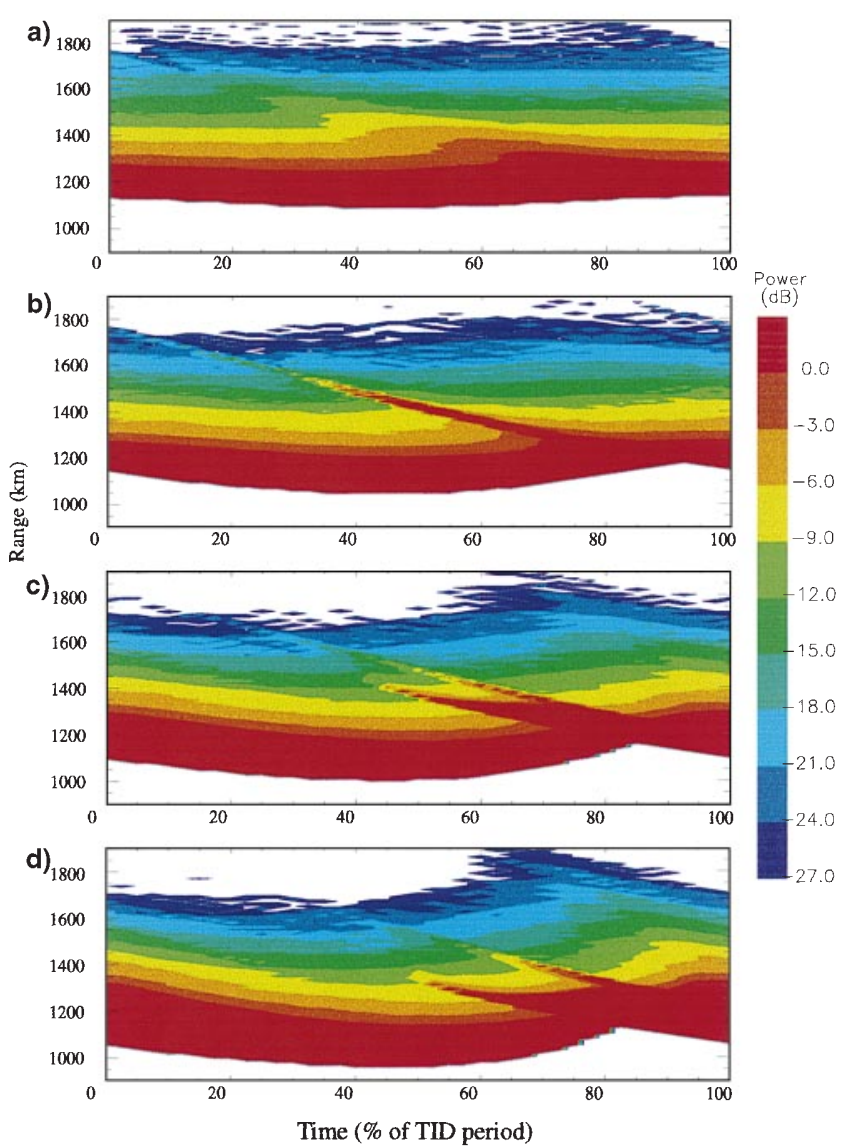

Fig. 4a-d. Range-time-intensity plots derived from ray tracing for the CUTLASS radar operating at $9.97 \mathrm{MHz}$ on 26 January 1997. TID electron density perturbation amplitude is a $5 \%$, b $15 \%$, c $25 \%$ and d $35 \%$

peak skip variation (i.e. the difference between maximum and minimum skip values in a complete TID period) has been plotted as a function of $\delta$, reveals that the skip variation and the asymmetry of the wave form increases with increasing $\delta$. The skip variation saturates at about $190 \mathrm{~km}$ for a radar frequency of $12.4 \mathrm{MHz}$ and at about $170 \mathrm{~km}$ at $9.97 \mathrm{MHz}$. This saturation can be contrasted with the variation of filament power with TID amplitude established by Bristow and Greenwald (1995) where, for realistic values of the perturbation amplitude, the power increases with increasing $\delta$.

\subsection{Skip distance for different radar beams}

As described earlier (Sect. 2) the CUTLASS radar can transmit in 16 different beam directions with azimuths ranging from $-36^{\circ}$ through to $+12^{\circ}$. The modelling results discussed in Sect. 3.3 have been repeated for each of the 16 radar beams and the combined results presented in Fig. 8. Here the contours represent the skip distance variation about a value of $1090 \mathrm{~km}$ in the case of a radar frequency of $9.97 \mathrm{MHz}$ and $1420 \mathrm{~km}$ for 12.4 MHz. As previously mentioned the model TID propagates from north to south, this direction roughly 

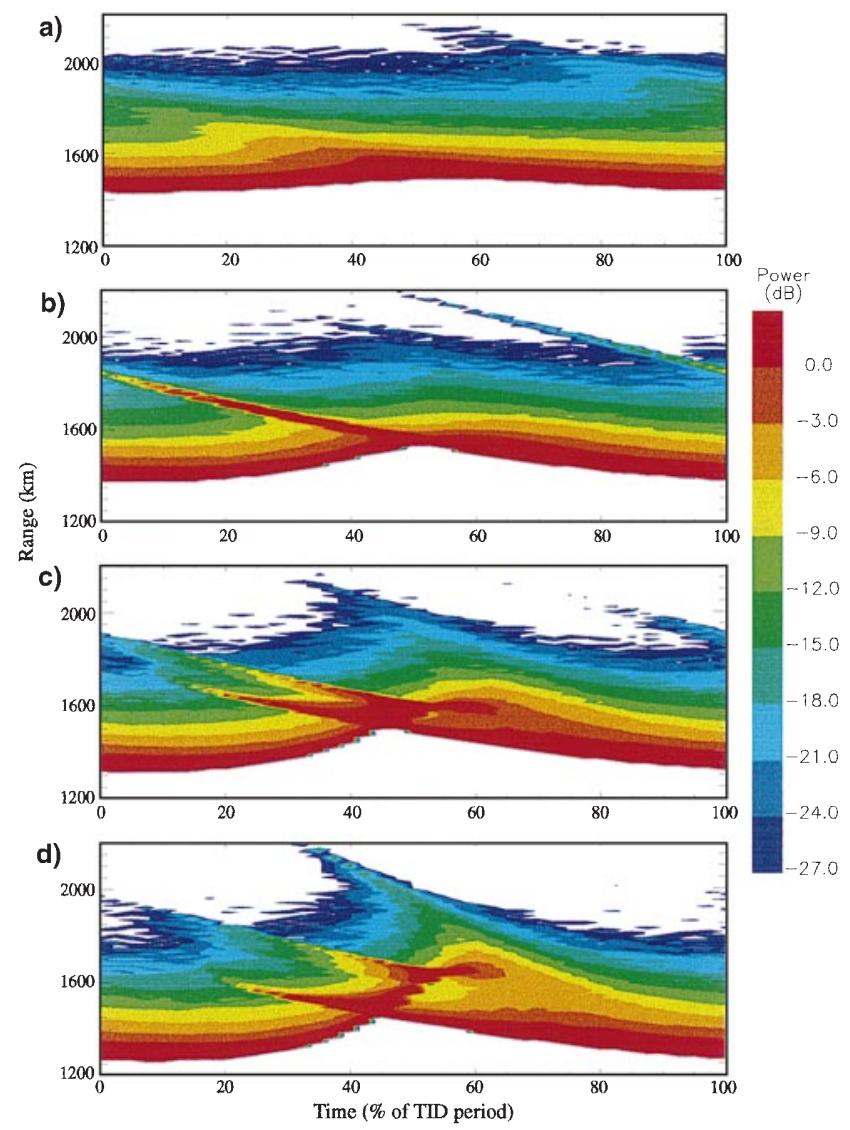

Fig. 5a-d. As for Fig. 4, except for a radar frequency of $12.4 \mathrm{MHz}$

corresponding to beam 11 of the radar. Figure 8 provides evidence that the peaks and troughs in the skip variation appear later in time in directions away from due north (i.e. beam 11). This effect is somewhat more pronounced for the higher radar frequency case. Furthermore, the relative skip variation is larger on beams well separated in azimuth from beam 11, the TID propagation direction.

\subsection{Skip variation for different frequencies}

Although the radar only transmitted on two frequencies during the experiment reported here, clearly the effect of employing other frequencies can be investigated by the ray tracing model as a guide to future experimentation. In Fig. 9, the skip variation and the relative signal strength at ranges close to the skip have been plotted as functions of radar frequency. While the skip variation increases as a function of frequency, the signal intensity decreases by nearly $30 \mathrm{~dB}$ going from a frequency of $10 \mathrm{MHz}$ to one of $18 \mathrm{MHz}$.

\section{Discussion}

A comparison of the range time intensity plots obtained by observation (Fig. 2) and those derived from model
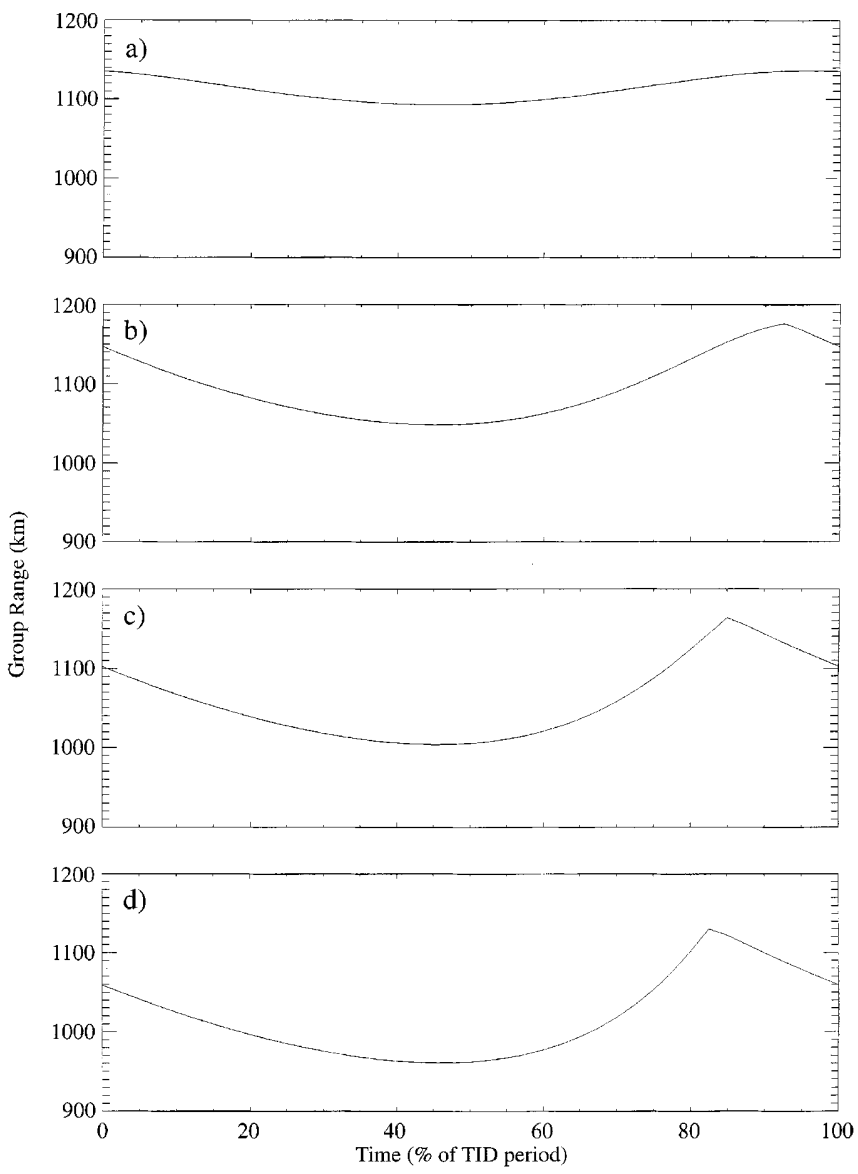

Fig. 6a-d. The skip variation for a radar frequency of $9.97 \mathrm{MHz}$ derived from ray tracing. The perturbation amplitude is a $5 \%, \mathbf{b} 15 \%$, c $25 \%$ and d $35 \%$. With no perturbation the skip is at $1115 \mathrm{~km}$

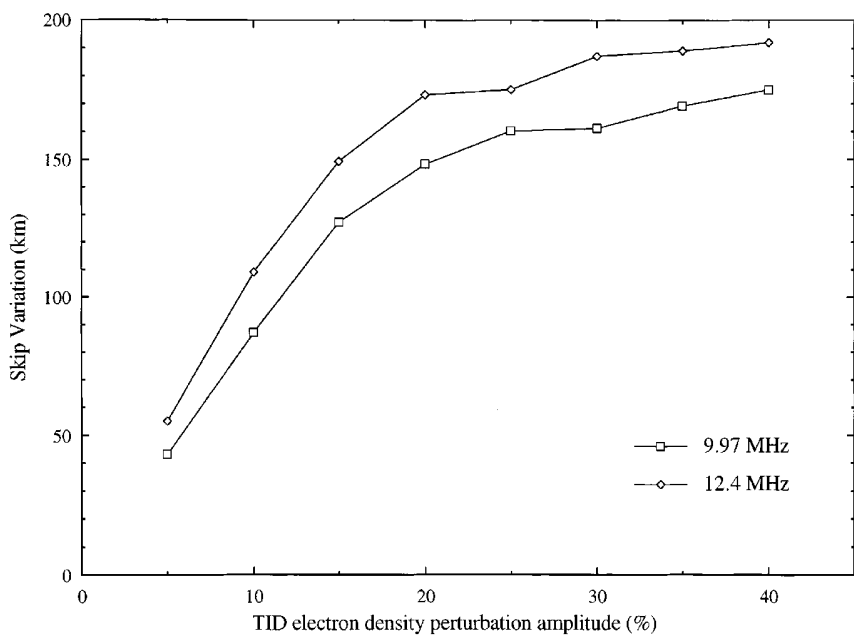

Fig. 7. The modelled skip variation as a function TID perturbation amplitude for two radar frequencies

(Figs. 4 and 5) reveals a number of similarities. Firstly, the filaments (regions of strong back-scatter which decrease in range with time) are present in both experimental and modelled results. These have been discussed extensively by Bristow and Greenwald (1995) 


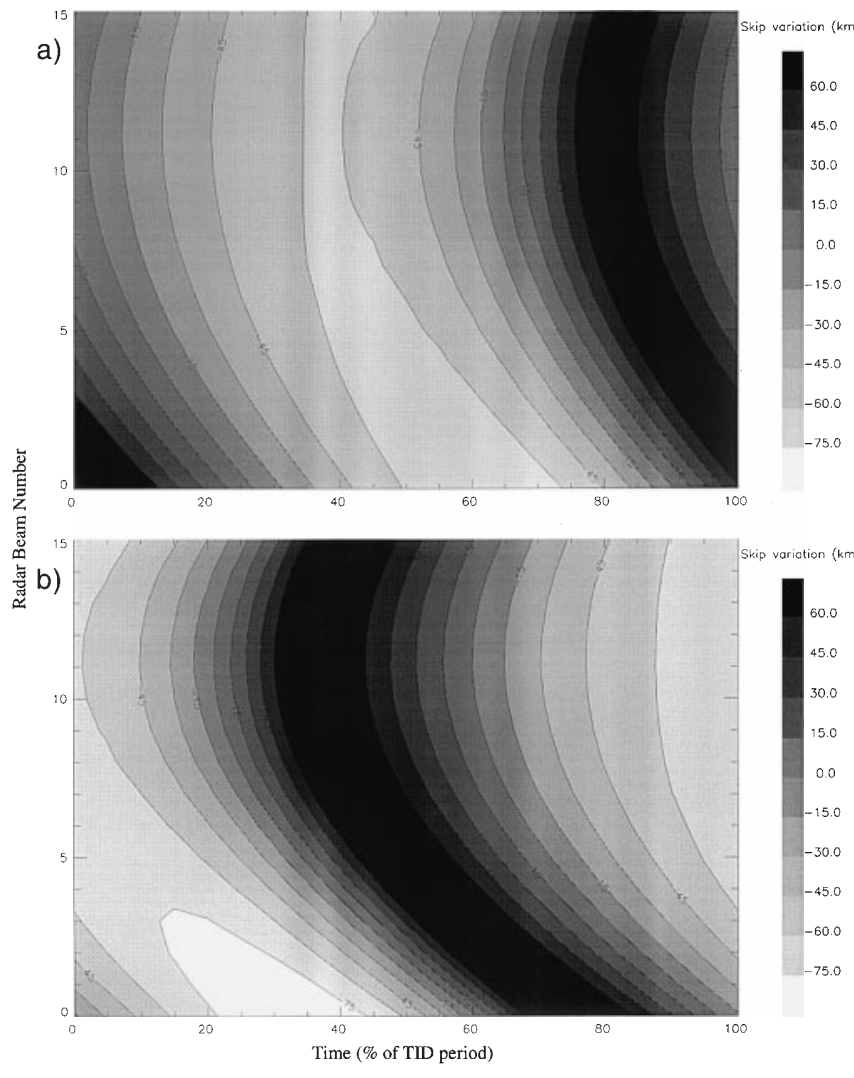

Fig. 8a, b. The modelled skip variation as a function of time and radar beam number (i.e. azimuth) for radar frequencies of a $9.97 \mathrm{MHz}$ and b $12.4 \mathrm{MHz}$. Note that zero corresponds to a skip of $1090 \mathrm{~km}$ in $\mathbf{a}$ and $1420 \mathrm{~km}$ in $\mathbf{b}$. The parameters used to model the TID are given in Table 2

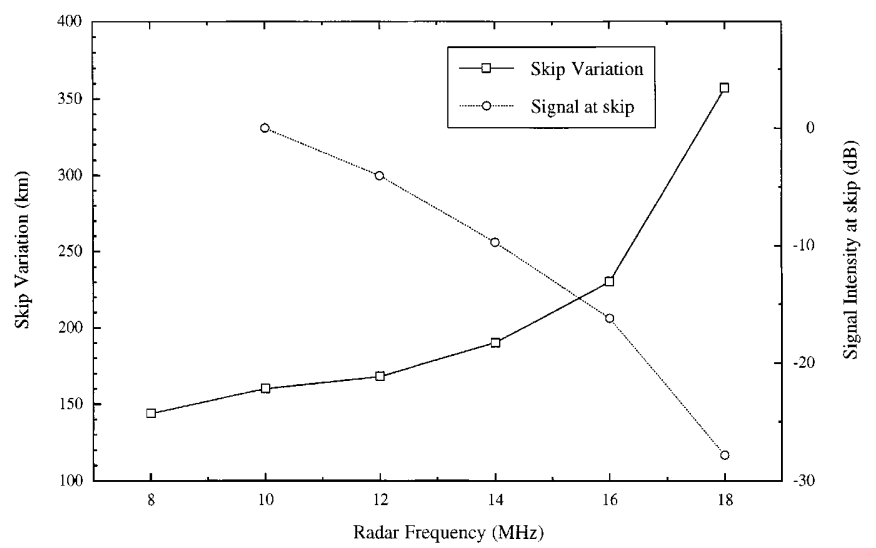

Fig. 9. The modelled skip variation and signal power returned from ranges within $90 \mathrm{~km}$ of the skip as a function of radar frequency

and can usually be considered a useful indicator of the presence of TIDs. It is interesting to note that sometimes the filaments are relatively weak even with strong TIDS (e.g. Fig. 2, $10 \mathrm{MHz}$ ) possibly as a result of the filament splitting found in the ray tracing results (Figs. 4 and 5). Secondly, both experiment and the modelling results exhibit a quasi-periodic variation in the skip distance which is attributable to the passage of a TID. It is interesting to note that although a sinusoidal variation in electron density has been employed in the modelling (Eq. 3), the resultant variation in skip is symmetrical in neither time nor range, especially as the intensity of the TID is increased.

The skip variation caused by the TID measured experimentally was about $150 \mathrm{~km}$ for a radar frequency of $9.97 \mathrm{MHz}$ and $180 \mathrm{~km}$ at $12.4 \mathrm{MHz}$. By comparing these values with those obtained as a function of $\delta$ from the model an estimate of the TID electron density perturbation amplitude responsible for the experimental observations can be found. In this case it appears that a perturbation amplitude of about $25 \%$ gives a skip variation consistent with observations. This value is consistent with those obtained from ionosonde measurements (e.g. Morgan, 1990) and with those deduced from HF radar data by Bristow and Greenwald (1995). However, the technique employed here, i.e. deriving the TID perturbation amplitude from skip measurements, has several significant advantages over the method adopted by Bristow and Greenwald (1995), where they essentially examined the relative amplitude of the filament. These advantages are firstly that the radar ground reflected back-scatter returns are strongest close to the skip and hence it is possible that data will be available from these ranges when perhaps the filament is not visible. It is also clear from Figs. 4 and 5 that at high values of $\delta$ more than one filament can be present potentially making identification difficult. Furthermore, when $\delta$ is below about $10 \%$ the filaments may not be present meaning that weak TIDs cannot easily be identified by this method. Secondly, the amplitude of the signals are reliant on a number of factors, for instance, knowledge of the $\mathrm{D}$ region absorption, and hence errors in the interpretation of the data may occur while the determination of $\delta$ from the skip measurements relies only on refractive effects in the F-region. However, there are potentially several limitations of the skip-variation technique. Firstly, during the modelling reported here the effect of the E region has been ignored. With a strong $E$ region it is possible that the ranges near the $\mathrm{F}$ region skip could be contaminated by $\mathrm{E}$ region back-scatter (although this would also affect results obtained by the method of Bristow and Greenwald 1995). Secondly, the range resolution employed by the radar in these experiments was $45 \mathrm{~km}$ while the skip variation was around $160 \mathrm{~km}$ (i.e. 4 range gates). Future experiments will utilise a range resolution of $15 \mathrm{~km}$. Next, the modelling results predict that the skip variation saturates at TID intensities of about $30 \%$ and hence TIDs larger than this cannot be distinguished. However, it is likely that TIDs with intensities significantly greater than $30 \%$ are comparatively rare so this may not be an important limitation. Lastly in the absence of radar returns at ranges further than the skip, and therefore no filaments, it may become difficult to identify the presence of TIDs solely from the skip variation. However, a variation in the skip which appears in all 16 radar beams and which has a period similar to those of a mediumscale gravity-wave would be unlikely to be generated by any other means. For multiple TIDs with significantly 
different periods, the filtering of the data will discriminate between the two. When the periods are similar but the sources are widely separated then the presence of the two TIDs will become evident in the phase front propagation across the 16 radar beams. However, if the two sources are close to each other and have a similar period then it will not be possible to resolve them.

Figures 3 and 8 , where the skip variation has been plotted as a function of beam number (i.e. azimuthal direction) and time, display one advantage of employing an HF coherent radar to investigate TIDs, namely the wide spatial coverage compared to either an ionosonde network or incoherent scatter radars. Firstly it should be pointed out that the beams transmitted from the CUTLASS, Finland station are arranged such that beam 15 has an azimuth $\sim 12^{\circ} \mathrm{E}$, beam 11 points almost due north, and beam $0-36^{\circ} \mathrm{W}$ (Fig. 1). Examination of the model data in Fig. 8 yields the information that features in the data (e.g. peaks or troughs) occur earlier when the radar is looking anti-parallel to the direction of TID travel. Interpretation of the observations (Fig. 3) then implies that prior to about 1230 UT the TIDs were travelling from a source somewhat to the east of the radar field-of-view, while by 13 UT or so, the line of travel lay somewhere within the field-of-view. However, it must also be remembered that the model assumes a uniform ionosphere across the entire field of view which the F-region critical frequencies measured at different ionosonde stations (Table 1) indicate is certainly not the case either prior to about $10 \mathrm{UT}$ or after $13 \mathrm{UT}$. Therefore, it may be possible that the apparent change in source location is a result of changes in $\mathrm{HF}$ propagation conditions and so a more precise determination of the TID motion has not yet been obtained.

The ray-tracing results for different radar frequencies provide an opportunity to optimise the design of future experiments. The results in Fig. 9 illustrate that a compromise is required, namely that as the radar frequency is increased the skip variation also increases, thereby improving the ability of the radar to observe such variation. However, as the frequency is increased then the signal amplitude of the returns close to the skip is reduced.

\section{Conclusions}

The effects of travelling ionospheric disturbances (TID) on the range of the closest ground scattered returns measured by the Finland station of the CUTLASS radar have been studied both experimentally and by a ray tracing simulation. The results of the simulation indicate that the variation in skip caused by the passage of a TID increases as the intensity of the TID increases up to an intensity of about $25 \%$. This property has been used to estimate the intensity of the TIDs observed by CUTLASS resulting in a value of about $25 \%$. It is considered that this method holds several advantages over that adopted by Bristow and Greenwald (1995) since the returns at the skip are relatively easy to detect and this distance is only determined by the refractive properties of the ionosphere.

A preliminary simulation of the effect of changing the radar frequency has indicated that the skip variation caused by the TID increases with increasing radar frequency for a fixed TID electron density perturbation amplitude. Since the best range resolution achievable by the CUTLASS radar is $15 \mathrm{~km}$ then increasing the frequency provides the best opportunity for detecting even quite weak TIDs. The results of a multi-frequency CUTLASS experiment will form the subject of a future paper.

Acknowledgements. The Ionosonde measurements were made available by the Sodankylä Geophysical observatory, the World Data centre (Kiruna) and EISCAT (Tromsø). The CUTLASS radars are deployed and operated by the University of Leicester, and are jointly funded by the UK Particle Physics and Astronomy Research Council, the Finnish Meteorological Institute and the Swedish Institute for Space Physics. One of us (N.A.) was supported by PPARC grant PPA/G/0/1997/000254.

The Editor-in-chief thanks G. Sozko and V. A. Bristow for their help in evaluating this paper.

\section{References}

Arnold, N. F., T. B. Jones, T. R. Robinson, A. J. Stocker, and J. A. Davies, Validation of CUTLASS HF radar gravity wave observing capability using EISCAT CP-1 data, Annales Geophysicae, 16, 1392-1399, 1998.

Bilitza, D., International reference Ionosphere 1990, edited by D. Bilitza, NSSDC 90-22, Greenholt, MA, USA, 1990

Bristow, W. A., and R. A. Greenwald, Estimating gravity wave parameters from oblique high-frequency backscatter: modeling and analysis, J. Geophys. Res., 100, 3639-3468, 1995.

Chimonas, G., and C. O. Hines, Atmospheric gravity waves launched by auroral currents, Planet. Space Sci., 18, 565-582, 1970.

Davies, K., Ionospheric radio, Peter Peregrinus, London, 1990.

Francis, S. H., A theory of medium-scale traveling ionospheric disturbances, J. Geophys. Res. 79, 5245-5260, 1974.

Georges, T. M., HF Doppler studies of travelling ionospheric distubances, J. Atmos. Terr. Phys., 30, 735-746, 1968.

Hines, C. O., Internal atmospheric gravity waves at ionospheric heights, Can. J. Phys., 38, 1441-1481, 1960.

Hocke, K., and K. Schlegel, A review of atmospheric gravity waves and travelling ionospheric disturbances: 1982-1995, Annales Geophysicae, 14, 917-940, 1996.

Hunsucker, R. D., Atmospheric gravity waves generated in the high-latitude ionosphere: a review, Rev. Geophys. Space Phys., 20, 293-315, 1982.

Jones, R. M., and J. J. Stephenson, A versatile three-dimensional ray tracing computer program, Office of Telecommunications Report 75-76, US Government Printing Office, Washington DC 20402, USA, 1975.

Kirchengast, G., K. Hocke, and K. Schlegel, Gravity waves determined by modeling of traveling ionospheric disturbances in incoherent-scatter radar measurements, Radio Sci., 30, 15511567, 1995.

Kirchengast, G., K. Hocke, and K. Schlegel, The gravity wave-TID relationship: insight via theoretical model-EISCAT data comparison, J. Atmos. Terr. Phys., 58, 233-243, 1996.

Milan, S. E., T. B. Jones, T. R. Robinson, E. C. Thomas, and T. K. Yeoman, Interferometric evidence for the observation of ground backscatter originating behind the CUTLASS coherent HF radar, Annales Geophysicae, 15, 29-39, 1997.

Millward, G. H., A resonance effect in AGWs created by periodic recurrent bursts in the auroral electric field, Annales Geophysicae, 12, 94-96, 1994. 
Morgan, M. G., Daytime travelling ionospheric disturbances observed at $\mathrm{L} \approx 4.5$ in western Quebec with rapid-run ionosondes, Radio Sci., 25, 73-83, 1990.

Munro, G. H., Travelling ionospheric disturbances in the F region. Aust. J. Phys., 11, 91-112, 1958.

Robinson, T. R., Acoustic gravity wave growth and damping in convecting plasma, Annales Geophysicae, 12, 210-219, 1994.
Samson, J. C., R. A. Greenwald, J. M. Ruohoniemi, A. Frey, and K. B. Baker, Goose Bay radar observations of Earth-reflected atmospheric gravity waves in the high-latitude ionosphere. J. Geophys. Res., 95, 7693-7709, 1990.

Shand, B. A., S. E. Milan, T. K. Yeoman, P. J. Chapman, D. M. Wright, T. B. Jones and L. T. Pedersen, CUTLASS HF radar observations of the Odden ice tongue, Annales Geophysicae. 16, 280-282, 1998. 